$\begin{array}{cl}\begin{array}{c}\text { Revue } \\ \text { de /histoire }\end{array} & \text { Revue de l'histoire des religions } \\ \text { des religions } & \begin{array}{l}3 \mid 2010 \\ \text { Varia }\end{array}\end{array}$

\title{
Le corps de Dieu dans le judaïsme rabbinique ancien. Problèmes d'interprétation
}

The Body of God in Ancient Rabbinic Judaism: Problems of Interpretation

José Costa

\section{OpenEdition}

Édition électronique

URL : http://journals.openedition.org/rhr/7617

DOI : $10.4000 /$ rhr.7617

ISSN : 2105-2573

Éditeur

Armand Colin

Édition imprimée

Date de publication : 1 octobre 2010

Pagination : 283-316

ISBN : 978-2200-92657-7

ISSN : 0035-1423

Référence électronique

José Costa, « Le corps de Dieu dans le judaïsme rabbinique ancien. Problèmes d'interprétation », Revue de l'histoire des religions [En ligne], 3 | 2010, mis en ligne le 01 octobre 2013, consulté le 19 avril 2019. URL : http://journals.openedition.org/rhr/7617 ; DOI : 10.4000/rhr.7617

Ce document a été généré automatiquement le 19 avril 2019

Tous droits réservés 


\section{Le corps de Dieu dans le judaïsme rabbinique ancien. Problèmes d'interprétation}

The Body of God in Ancient Rabbinic Judaism: Problems of Interpretation

José Costa

1 Sur la question du corps de Dieu, le christianisme a une position a priori paradoxale. Il soutient l'idée de l'incarnation de Dieu dans le corps d'un être humain. En même temps, dans la littérature des Pères, Dieu est conçu comme un être invisible et fondamentalement immatériel ${ }^{1}$. Ce paradoxe trouve cependant son explication sur un plan philosophique mais aussi scripturaire. Concernant le premier aspect, les Pères sont imprégnés par la philosophie grecque. Or l'aristotélisme comme le platonisme s'entendent sur l'existence d'une divinité fondamentalement immatérielle ${ }^{2}$. Sur le plan scripturaire, le verset de Jn 1, 18 est particulièrement parlant : «Dieu, nul ne l'a jamais vu; un Dieu, fils unique, qui est dans le sein du Père, celui-là l'a fait connaître ${ }^{3}$. C'est parce que Dieu, le Père, est inconnaissable et ne peut être vu que le Fils a été envoyé. Le Dieu fils, qui est corporel, permet d'accéder à la connaissance du Dieu Père qui ne l'est pas ${ }^{4}$.

2 Le judaïsme, pour sa part, a la réputation de croire en un Dieu invisible. La preuve en est qu'il refuse toute représentation de la divinité, celle des divinités du polythéisme comme celle du Dieu unique : «(Verset 3) Tu n'auras pas d'autres dieux [...] (verset 4) tu ne feras pas pour toi d'image sculptée (pesel) ainsi que toute forme (temuna) qui est dans le ciel en haut et sur la terre en bas [...] (verset 5) Tu ne te prosterneras pas devant eux et tu ne les serviras pas...» (Ex 20, 3-5). Selon une lecture minimaliste du verset 4, seules les idoles sont interdites ${ }^{5}$, mais selon une lecture plus radicale, toute représentation est problématique, même si elle ne concerne en rien le culte idolâtre : « Rabban Gamliel avait une représentation des "formes" (demut șurot) de la lune dans sa chambre haute, sur une tablette et sur le mur [...] Il est enseigné dans une barayta : "qui est dans le ciel" (Ex 20,4) pour inclure (dans l'interdit de la représentation) le soleil et la lune, les étoiles et les astres $^{6} . .$. » La représentation, dont parle ici la Mishna, consiste en des diagrammes 
montrant les différentes phases de la lune. L'utilité halakhique de ces diagrammes est évidente et il est a priori peu raisonnable de voir en Rabban Gamliel un idolâtre masqué. Le Talmud, qui s'appuie sur une barayta, c'est-à-dire une tradition tannaïtique, non consignée dans la Mishna, estime cependant que le verset d'Ex 20, 4 interdit toute représentation plastique des astres, quelle que soit la finalité de cette représentation.

Les Pères de l'Église ont pourtant une tout autre vision du judaïsme. Selon Justin Martyr, les docteurs juifs conçoivent Dieu comme pourvu de mains, de pieds et de doigts ${ }^{7}$. Origène affirme que les juifs imaginent Dieu comme semblable à un être humain et ils fondent leur croyance sur le verset d'Is 66,1 . Certains chrétiens partageraient ces conceptions ${ }^{8}$. Basile le Grand demande au chrétien d'éloigner de son esprit toute conception corporelle de Dieu : ce sont les juifs qui lui attribuent une "forme" (morphên) ${ }^{9}$. Arnobe de Sicca concorde avec Basile. Ce sont les juifs et les sadducéens qui prêtent des formes (formas) à $D^{D i e u^{10}}$. Comment comprendre ces attestations des Pères ? Certains les écarteront, parce que les théologiens chrétiens connaissent mal le judaïsme et qu'ils en ont une vision négative. La notion d'un juif qui adhère à la corporéité divine serait la conséquence de l'attachement de ce même juif à la lettre et de son incapacité à saisir le sens spirituel de l'Écriture. Gedaliahu Stroumsa a cependant montré l'intérêt historique de ces témoignages, qu'il serait peu judicieux de considérer comme simplement polémiques ${ }^{11}$.

En fait, on confond trop souvent deux problématiques qui sont distinctes, celle de la nature de Dieu, visible ou invisible et celle de la représentation plastique de Dieu, permise ou interdite. Il est certes possible d'articuler les deux: s'il est interdit de représenter Dieu, c'est parce qu'il est invisible. Mais on peut aussi penser que Dieu ne peut être représenté parce qu'il a un corps, qui n'est semblable à aucun des corps existants dans notre monde. Dans le cadre du judaïsme, trois questions doivent être posées : 1) Dieu a-t-il un corps ? 2) En admettant que la réponse à la première question soit positive, ce corps peutil être vu? Il faut comprendre le verbe "pouvoir » dans ses deux acceptions : " pouvoir " au sens d'être capable de faire quelque chose et "pouvoir " au sens d'avoir le droit de faire quelque chose. La vision de Dieu peut être possible techniquement, sans être pour autant autorisée. Elle peut aussi être possible tout en comportant des dangers ; 3) Le corps de Dieu peut-il être représenté ? Le judaïsme ancien (biblique et rabbinique) a vraisemblablement répondu ainsi à ces trois questions : 1) Dieu a un corps ; 2) Ce corps peut être vu mais pas par n'importe qui et dans n'importe quelles circonstances ; 3) Il est interdit de représenter Dieu de manière plastique, sauf si la représentation est indirecte (sous la forme des chérubins de l'arche). Il est en revanche permis de le représenter dans un support verbal, oral ou écrit ${ }^{12}$.

5 La démonstration par Maïmonide de l'incorporéité divine n'est guère en harmonie avec le judaïsme rabbinique ancien qui, sur la question du corps divin, a plutôt trouvé son héritier dans la tradition kabbalistique. Mais, troisième principe de foi de Maïmonide, intégré comme tel dans le judaïsme ultérieur, l'incorporéité divine a frayé son chemin, y compris chez les kabbalistes ${ }^{13}$.

6 En dépit des obstacles nourris par la tradition rabbinique et perpétués en grande partie dans le monde de l'université, le fait que, pour le judaïsme rabbinique ancien, Dieu a un corps est de plus en plus souvent reconnu dans la recherche récente ${ }^{14}$. Aucune étude ne réunit cependant l'essentiel des matériaux pertinents, sources et commentaires. Un certain nombre de questions méritent aussi d'être placées au centre de l'attention du chercheur. Qu'est-ce qui autorise un commentateur ancien ou moderne à lire un texte corporéiste d'une manière métaphorique ? À quelles conditions une telle lecture est-elle 
légitime? Si les rabbins croient véritablement en un corps divin, cela oblige aussi à repenser leur rapport avec le paganisme « idolâtre » et le christianisme de l'incarnation.

\section{La Bible}

7 Le fait que Dieu a un corps apparaît nettement dans un grand nombre de versets. Il s'agit d'un corps à première vue semblable à celui de l'homme, d'où le terme justifié d'anthropomorphisme, à condition de l'employer dans un sens étroit, conforme à son étymologie. Le Dieu de la Bible a également des sentiments humains et l'on peut parler dans ce cas d'anthropopathisme ${ }^{15}$. Dans un ouvrage récent, Esther J. Hamori distingue cinq types fondamentaux d'anthropomorphisme : corporéiste concret, visuel, immanent, transcendant et figuratif ${ }^{16}$. Sans entrer autant dans les détails, nous nous limiterons à quelques versets particulièrement significatifs.

$8 \mathrm{Nb}$ 12, 8 affirme explicitement que Dieu a une forme (temurat ha-shem) et Ez 1, 26 qu'il est semblable à un homme : "et sur la ressemblance de trône une ressemblance, comme l'apparence d'un homme ». De nombreux versets attribuent à Dieu des organes du corps humain ${ }^{17}$. Dieu se fait également voir, notamment aux patriarches ${ }^{18}$. Certains versets évoquent la vision de Dieu sans pour autant préciser l'apparence de la chose vue, comme Ex 24, 10-11. Dt 4, 12 (et 15) rejette à première lecture l'idée d'un corps divin: «Et l'Éternel vous parla du sein du feu, une voix de paroles vous avez entendue et une forme ( temuna) vous n'avez pas vue, uniquement une voix.» On parle couramment sur cette question d'un courant de pensée deutéronomiste, proche de celui qui s'exprime dans le Deutéro-Isaïe (Is 40,18). Cette lecture est cependant bien loin d'aller de soi ${ }^{19}$. Dt 4, 12 et 15 affirment que les Hébreux n'ont pas vu Dieu au Sinaï, mais cela n'implique nullement que Dieu soit dénué de corps : ce corps a pu rester tout simplement dans un état d'occultation, du moins pour la grande masse des Hébreux. Gn 1, 26 constitue un autre exemple de verset ambivalent ${ }^{20}:$ «Et Dieu dit : Faisons l'homme à notre image (șalmenu) et comme à notre ressemblance (ki-demutenu).» Les termes șelem et demut ont un sens corporel très marqué. Șelem est même l'un des termes employés par la Bible pour désigner l'idole ${ }^{21}$. Le contexte incite cependant à ne pas écarter une lecture plus «spiritualiste ${ }^{22}$ » du verset, car le rédacteur du verset 26 juxtapose l'image et la ressemblance avec le fait que l'homme va dominer la création. N'est-ce pas dans la notion de souveraineté que se trouve l'élément commun à l'homme et à la divinitéé ${ }^{23}$ ? Ces deux lectures de Gn 1, 26-27 ne s'excluent d'ailleurs pas nécessairement l'une l'autre ${ }^{24}$.

Si l'on excepte donc quelques versets ambivalents, il ressort clairement de la lecture de la Bible que Dieu a un corps. On pourrait objecter à cela que tous les versets anthropomorphiques ne doivent pas être lus de manière littérale : ils constituent en fait des métaphores qui nous informent sur des aspects «spirituels» de la divinité. Cette lecture métaphorique des anthropomorphismes bibliques, qui paraît si naturelle à la plupart de nos contemporains et que l'on trouve chez les penseurs les plus éminents du judaïsme, se heurte cependant à un certain nombre de difficultés. La première de ces difficultés réside dans le postulat qui fonde cette lecture métaphorique et qui n'est pas toujours présenté de manière explicite: il est clair, par définition, que Dieu n'a pas de corps et il va donc de soi que tous les versets qui lui attribuent un corps doivent être lus de manière métaphorique. Il reste à se poser la question suivante : pourquoi Dieu serait-il par définition incorporel? Sur quel savoir s'appuie celui qui soutient cette opinion? Certains répondront à cette question en partant de la Bible elle-même. C'est parce que la 
Bible affirme explicitement que Dieu est incorporel qu'il faut relire à partir de ce fait tous les versets qui semblent affirmer le contraire. Cette réponse se heurte cependant à une importante difficulté : on ne trouve aucun verset biblique qui affirme explicitement que Dieu est incorporel $^{25}$. Les versets de Dt 4, 12 et 15 peuvent certes suggérer l'incorporéité de Dieu mais ils ne l'imposent en aucune manière. Certains objecteront alors : si la Bible ne donne aucune indication explicite dans le sens de l'incorporéité, il faut s'appuyer sur un savoir extérieur à la Bible et fiable. À la lumière de ce savoir, les versets trouveront leur véritable signification. Dire que Dieu a un corps serait une affirmation du même ordre que celle qui attribue une aile à la terre (Is 24,16$)$. Personne ne songe à prendre au pied de la lettre l'expression « l'aile de la terre ", car la science permet de connaître la terre et nous donne l'assurance que celle-ci n'a pas d'aile. C'est un savoir extérieur à la Bible, à caractère scientifique, qui permet de préciser le sens de l'expression biblique «l'aile de la terre ». Il est possible de raisonner de même dans la question du corps divin, ce que fait Maïmonide dans le Guide des Perplexes. La science par excellence qu'est la philosophie aristotélicienne démontre l'incorporéité de Dieu et c'est l'existence de cette certitude scientifique qui permet de lire les versets corporéistes de manière métaphorique ${ }^{26}$. L'intervention d'un savoir extérieur à celui de la Bible a cependant été critiquée, à juste titre, du côté des théologiens comme du côté des rationalistes ou des hommes de science. Certains penseurs juifs estiment que l'introduction de la philosophie grecque dans la lecture de la Bible est une trahison du sens originel de la révélation ${ }^{27}$. Les hommes de science, pour leur part, partent du principe qu'un texte doit d'abord être compris à partir de lui-même, selon ses propres catégories ${ }^{28}$.

10 Un dernier facteur doit intervenir dans la question de l'interprétation des textes corporéistes de la Bible, celui de la tradition talmudique. Les rabbins de l'Antiquité prétendent avoir des traditions, contenues dans la Tora orale, qui leur permettent de mieux comprendre un certain nombre de versets problématiques. En s'appuyant sur leurs traditions et sur les indications données par Dt 4, 12 et 15, ont-ils interprété les versets corporéistes dans un sens métaphorique?

\section{L'interprétation par les rabbins des versets corporéistes}

11 Le rapport des rabbins aux anthropomorphismes bibliques a été étudié de manière globale dans un ouvrage d'Arthur Marmorstein ${ }^{29}$. Celui-ci couvre non seulement le problème du corps divin (anthropomorphisme) mais aussi celui des émotions humaines prêtées à Dieu (anthropopathisme). Selon A. Marmorstein, les rabbins sont divisés en deux courants de pensée. Certains lisent les versets anthropomorphiques et anthropopathiques de manière littérale, d'autres leur donnent une signification métaphorique. Pour les premiers, Dieu a un corps, pour les autres, il en est dénué. Ce débat est particulièrement marqué à l'époque des tanna'im, entre les écoles de Rabbi 'Aqiba (lecture littérale/anthropomorphisme) et Rabbi Yishma'el (lecture métaphorique/ antianthropomorphisme). À l'époque plus tardive des amora'im, le courant littéraliste/ anthropomorphique l'emporte ${ }^{30}$. Ces conclusions d'A. Marmorstein ont été partiellement remises en question par la recherche récente. Selon Alon Goshen Gottstein, le débat entre les deux courants n'existerait qu'au niveau des anthropopathismes. Les rabbins seraient en revanche tous d'accord pour concevoir un Dieu corporel, comme en témoigne leur lecture systématiquement corporéiste des versets de Gn 1, 26-27, Gn 5, 3 et Gn 9, 631. Il 
n'est donc pas sans intérêt de s'arrêter quelque peu sur l'interprétation rabbinique de ces versets.

Les versets de Gn 1, 26-27 sont assez peu commentés dans le corpus rabbinique ancien. Le verset de Gn 9, 6 a fait l'objet d'un plus grand nombre de commentaires. Dans les commentaires qui sont consacrés à $G n$ 1, 26-27, le rabbin évite presque systématiquement de préciser le sens des mots "image» et "ressemblance», préférant tourner son attention vers d'autres aspects de ces deux versets. Le pluriel « faisons » est par exemple souvent commenté ${ }^{32}$. Selon A. Goshen Gottstein, Gn 9, 6 est plus commenté parce qu'il a un contenu pratique, dont $\mathrm{Gn}$ 1, 26-27 est dépourvu ${ }^{33}$. Les rabbins, toujours soucieux de la halakha, mettraient l'accent sur le verset qui est le plus susceptible d'avoir des implications concrètes. Nous avons vu plus haut que Gn 1, 26-27 est ambivalent. Par les termes employés (" image », " ressemblance »), il fait plutôt référence au corps mais par le contexte immédiat (la question de la domination de l'homme sur la nature), il fait référence à "l'esprit». Gn 5, 3 et Gn 9, 6 participent indirectement de la même ambivalence. Ces versets sont donc une excellente occasion de tester la validité du modèle d'A. Marmorstein. S'il y a bien, comme le dit A. Marmorstein, deux courants de pensée et deux écoles sur la question du corps de Dieu, il devrait y avoir deux lectures possibles de ces versets, qui se prêtent particulièrement à cette dualité, par leur ambivalence même.

Voici quelques exemples de commentaires explicites ou implicites des versets de Gn 1, 26-27, Gn 5,3 et Gn 9, 6, où la question de l'image et de la ressemblance est abordée :

«Hillel l'Ancien, alors qu'il marchait avec ses disciples, s'apprêta à prendre congé d'eux. Ses disciples lui demandèrent: Où vas-tu? Il leur répondit: Faire un commandement. (Ils dirent:) Et quel commandement Hillel va-t-il faire? Il leur répondit: (Celui) de me laver dans un bain. Ils lui dirent: Et cela est un commandement! Il leur répondit: Oui! Si (pour) les statues des rois (iqonin shel melakhim) que l'on dresse dans leurs théâtres et dans leurs cirques, celui qui est préposé à les nettoyer et à les laver, on l'honore avec un turban, et ce n'est pas tout car il est honoré avec les grands du royaume, moi qui suis créé à l'image et à la ressemblance, ainsi qu'il est dit : "Car à l'image de Dieu, il a fait l'homme" (Gn 9, 6), combien plus ${ }^{34}$ !»

« Rab Huna fils de Rab Yehoshua' a dit: A la session d'étude d'Abbaye, j'ai entendu que (le verset) "Vous ne ferez pas avec moi (itti) (des dieux d'argent et des dieux d'or)" (Ex 20, 23) (signifie en fait) "vous ne ferez pas de moi (oti)" "35."

«L'Écriture rapporte: "Je suis l'Éternel, ton Dieu" $(E x ~ 20,2)$ et en face de ce (commandement), il est écrit: “Tu ne tueras pas" (Ex 20, 13). L'Écriture rapporte que toute (personne) qui verse le sang, on la considère comme si elle avait diminué la ressemblance (demut) du roi. Parabole d'un roi de chair et de sang qui entra dans une ville, on dressa pour lui des statues (iqonot), on lui fit des images (șelamim) et on frappa pour lui des monnaies (à son effigie). Plus tard, ils renversèrent les statues, cassèrent ses images, rejetèrent ses pièces et diminuèrent la ressemblance du roi ${ }^{36}$ .»

"S'il en est ainsi, quelle est la signification (ma talmud lo-mar) de "un homme intègre et droit" (Jb 1, 1) ? En fait, (le verset nous) enseigne que Job est né circoncis. Le premier homme est aussi sorti circoncis, ainsi qu'il est dit: "Et Dieu créa l'homme à son image" (Gn 1, 27). Seth est aussi sorti circoncis, ainsi qu'il est dit : "Et il engendra, comme à sa ressemblance, à son image" $(\mathrm{Gn} 5,3)^{37}$. "

14 Dans le premier texte, Hillel l'Ancien présente à ces disciples le fait de se laver comme un commandement. En effet, celui qui, dans la parabole, lave les statues du roi, est semblable à l'homme qui lave son propre corps. La création de l'homme "à l'image et à la ressemblance » signifie donc qu'il est pourvu d'un corps semblable à celui de Dieu. Dans le 
deuxième texte, Rab Huna estime qu'on ne peut pas lire de manière littérale le verset d'Ex 20, 23 : « Vous ne ferez pas avec moi (itti) (des dieux d'argent et des dieux d'or). » Lire le verset dans sa littéralité impliquerait que l'interdit ne porte que sur le syncrétisme : on ne peut adorer une idole de Dieu avec des statues d'autres divinités et donc il serait autorisé d'adorer une idole de Dieu si elle est unique! Rab Huna propose alors de lire : «vous ne ferez pas de moi (oti) ». Mais cette lecture pose aussi un problème : pourquoi insister sur « de moi »? Il aurait suffi de dire : «Vous ne ferez pas des dieux d'argent et d'or » et on aurait compris une double interdiction, celle de représenter Dieu et celle de représenter les autres dieux. C'est pourquoi le « de moi » ne porte pas directement sur Dieu lui-même mais sur ce qui ressemble à Dieu: l'homme. Le verset interdit donc la représentation de l'homme, parce qu'il est à l'image de Dieu, ou pour être plus précis, il interdit la représentation du corps de l'homme parce qu'il est à l'image du corps de Dieu. Dans le troisième texte, la parabole décrit un phénomène historique bien connu, celui de la damnatio memori ${ }^{38}$. Quand on veut souiller le souvenir du roi, on détruit ses représentations. Le roi correspond à Dieu, la statue du roi n'est autre que l'homme et donc détruire la statue du roi équivaut à tuer l'homme. L'image de Dieu dans l'homme («la ressemblance du roi ») inclut nécessairement le corps dans ce contexte, car le meurtre affecte d'abord et surtout l'enveloppe corporelle de l'être humain. Dans le quatrième texte, la ressemblance entre Dieu et l'homme est un élément corporel précis : la circoncision. Dieu a donc non seulement un corps mais celui-ci est conçu comme circoncis.

\section{Le corps lumineux}

Dans un certain nombre de textes, qui ne sont pas nécessairement liés à l'exégèse des versets de Gn 1, 26-27, Gn 5, 3 et Gn 9, 6, le corps divin revêt une autre caractéristique singulière : il est de nature lumineuse. A. Goshen Gottstein a particulièrement insisté sur ce point dans son article, même s'il est loin d'avoir épuisé tous les aspects de la question. On trouve dans le corpus rabbinique trois formulations directes de la notion de corps divin lumineux et une formulation indirecte. Les trois formulations directes sont: la gloire, la Shekhina et le manteau. Le terme de "gloire " désigne une manifestation sensible de Dieu ${ }^{39}$. Il est déjà employé en ce sens dans la Bible. Dans 1 R 8, 10-11, la gloire équivaut à une nuée obscure. Dans d'autres versets, elle a au contraire un caractère lumineux

(Ex 24, 17 ; Ez 43, 2). Les rabbins évoquent la «mesure de la gloire» ou la «forme de la gloire $^{40}$ ». La deuxième formulation est typiquement rabbinique : il s'agit de la Shekhina, même si elle est parfois associée au terme biblique "gloire ${ }^{41}$ ». À la base, le terme de Shekhina n'a rien de lumineux, puisqu'il provient du verbe shakhan qui signifie résider et que l'on trouve dans le mot mishkan, le tabernacle ${ }^{42}$. Les rabbins évoquent souvent l'éclat de la Shekhina, dans le cadre du Sinaï ou dans celui du monde futur. Le texte suivant a l'avantage d'associer les deux contextes :

« (Voici) une phrase de la bouche de Rab: Il ne (sera) pas comme le monde présent, le monde futur! Le monde futur, il n'y aura en lui ni nourriture, ni boisson, ni sexualité, ni jalousie, ni haine, ni dispute, mais les justes (seront) assis, leurs couronnes sur leurs têtes et ils se nourriront de l'éclat de la Shekhina, ainsi qu'il est dit : "Ils virent Dieu, ils mangèrent et ils burent" $(\mathrm{Ex} 24,11)^{43}$."

La doctrine du manteau dérive directement d'un verset biblique, Ps 104, 2 : «Tu te revêts de la lumière comme un manteau, tu déploies les cieux comme une tente. » La deuxième 
partie du verset, sur le déploiement des cieux, fait référence aux débuts de la création. La création du ciel (et de la terre) aurait donc été opérée à partir de la lumière d'un mystérieux manteau, dont parle la première partie du verset :

«Rabbi Shim'on ben Yehoṣadaq demanda à Rabbi Shemu'el bar Nahmani en lui disant : Parce que j'ai entendu sur toi que tu es un maître dans la aggada, (dis-moi) d'où la lumière a été créée ? Il lui répondit : (Cela nous) enseigne que le Saint, béni soit-Il, s'est enveloppé en elle comme dans un manteau et il a fait briller l'éclat de sa splendeur (hibhiq ziw hadaro) d'une extrémité du monde à l'autre. Il la lui a dite (cette tradition) dans un murmure. Il lui dit (alors) : Il y a un verset explicite (à ce sujet) : "Tu te revêts de la lumière comme un manteau" (Ps 104, 2) et toi, tu me (la) dis dans un murmure, c'est étonnant! Il répondit: De même que je l'ai entendue dans un murmure, de même je te la dis dans un murmure ${ }^{44}$."

17 Le texte semble décrire un double processus d'émanation. Dieu commence par s'envelopper dans une lumière préexistante ( le Saint, béni soit-Il, s'est enveloppé en elle comme dans un manteau »), ce qui lui permet ensuite d'émaner une autre lumière («il a fait briller l'éclat de sa splendeur d'une extrémité du monde à l'autre »). Il est possible qu'il y ait un rapport entre cette double émanation et la lumière de la Shekhina.

L'idée d'un corps divin lumineux connaît aussi une formulation indirecte, à laquelle A. Goshen Gottstein donne une place considérable, celle de l'Adam lumineux ${ }^{45}$. Selon Rabbi Shim'on ben Menasya, "si la "pomme" du talon du premier homme assombrissait la sphère du soleil, combien plus le rayonnement de sa face ${ }^{46} »$. Pour Rabbi Me'ir, les tuniques de peau (kotnot 'or) dont parle Gn 3, 21 sont en fait des tuniques de lumière ( kotnot or $)^{47}$. Si l'image et la ressemblance que partagent Dieu et Adam sont bien de naturelle corporelle et qu'Adam est doté d'un corps lumineux, il en découle que Dieu lui aussi est doté d'un corps de cette nature.

L'affirmation la plus explicite du corps lumineux divin n'est pas dans la littérature rabbinique mais dans un texte judéo-chrétien des Homélies Pseudo-clémentines: «Car il a une forme [...] il a tous les membres mais non pour s'en servir [...] son corps étant incomparablement plus lumineux que l'esprit par lequel nous voyons ${ }^{48}$.» Ce texte constitue le deuxième pilier de la démonstration d'A. Goshen Gottstein ${ }^{49}$. Il n'est pas sans intérêt que les judéo-chrétiens disent plus directement ce que les rabbins laissent souvent plutôt entendre. Le judaïsme rabbinique serait-il plus ésotérique et plus normatif que son rival judéo-chrétien ${ }^{50}$ ?

\section{La vision de Dieu}

La doctrine du corps divin et celle de la vision de Dieu entretiennent des liens inévitables. Il ne peut y avoir vision de Dieu que si Dieu a une apparence sensible. La vision de Dieu intervient dans deux contextes différents, celui de l'exode et celui du monde futur, comme dans le cas de la Shekhina, évoqué plus haut. Concernant la vision au moment de l'exode, c'est-à-dire au bord de la Mer Rouge et au mont Sinaï, les divergences des rabbins sont nombreuses. Selon certains, seul Moïse a vu Dieu ${ }^{51}$. Selon d'autres, cette vision a été le bien de tout Israël ${ }^{52}$. Même le cas de Moïse ne fait pas l'unanimité. Sa vision n'aurait pas été pleine et entière (mar'e shekhina) mais un degré inférieur de vision (mar'e dibbur) ${ }^{53}$. Certains affirment que Moïse n'a jamais vu la face de Dieu en ce monde ${ }^{54}$. Ces divergences recoupent en partie celles qui se manifestent déjà dans le texte de la Tora ${ }^{55}$. Il est cependant remarquable que même les traditions qui privent Israël, voire Moïse, de la 
vision de Dieu, ne nient pas que Dieu ait un corps. Le texte suivant de la Mekhilta nous présente une conception de la vision de Dieu, à la fois très directe et démocratique :

" "Celui-ci est mon Dieu (et je veux le glorifier)" (Ex 15, 2). Rabbi Eliezer dit: D'où (peux)-tu dire qu'une servante a vu au bord de la Mer (Rouge) ce que n'a pas vu Isaïe ainsi qu'Ezéchiel ? car il est dit : “Et par la main des prophètes, j’ai révélé ma forme (adamme)" (Os 12,11) et il est écrit: "Les cieux s'ouvrirent et j'ai vu (des visions divines)" (Ez 1, 1) $)^{56}$."

Dans le verset d'Ex 15, 2, Rabbi Eliezer commente le démonstratif « celui-ci » : il prouve que Dieu était vraiment devant les Hébreux, au point qu'il était possible de le montrer du doigt ${ }^{57}$. Les deux autres versets cités témoignent au contraire d'une vision moins directe. Os 12, 11 emploie le verbe adamme, qui provient de la même racine DMH que le substantif demut, « ressemblance ». Il signifie donc très précisément : «J'ai révélé quelque chose qui ressemble à ma forme ». Quant au verset d'Ez 1, 1, il dit qu'Ezéchiel a "vu des visions divines », ce qui sous-entend qu'il n'a pas vu Dieu lui-même. Le terme "vision» (mar'e) signifie aussi «apparence » et l'on sait que l'apparence n'est pas toujours conforme à ce qu'est la chose elle-même.

La vision est parfois présentée comme un objet de désir, de la part du peuple d'Israël. La dimension corporelle voire érotique de cette vision n'en ressort que plus nettement :

"Autre interprétation de "afin que (le peuple entende quand je te parlerai)" (Ex 19, 9). Ils ont dit: Nous voulons voir le roi (car) celui qui entend n'est pas semblable (lo dome) à celui qui voit. Le Lieu a dit: Donne-leur ce qu'ils demandent, "car le troisième jour, l'Éternel descendra (aux yeux de tout le peuple sur le Mont Sinaï)" $(\operatorname{Ex} 19,11)^{58} . »$

\section{Le corpus mystique juif de l'Antiquité}

Le corpus mystique est celui de la littérature du char (merkaba) ou des palais (hekhalot) et celui du Shitur Qoma, «mesure de la taille » qui appartient en partie au corpus précédent. Les textes du Shi'ur Qoma sont les plus explicites dans l'attribution d'un corps à Dieu. Ils en décrivent les mensurations précises qui sont gigantesques ${ }^{59}$. Il est possible que le Shitur Qoma constitue, à la base, un commentaire du Cantique des Cantiques ${ }^{60}$. Il contiendrait la description physique de l'amant divin qu'Israël a rencontré au Sinaï. Dans la littérature de la merkaba, le mystique fait l'ascension des différents cieux ou palais célestes, dans le but de voir le char divin, la merkaba ainsi que la forme humaine qui se trouve sur le trône (Ez 1, 26). Certains textes du corpus de la merkaba contiennent des matériaux qui se rattachent au Shi'ur Qoma ${ }^{61}$. D'autres évoquent l'apparence de Dieu sans être aussi précis que le Shi ur Qoma ${ }^{62}$. Ils mentionnent, par exemple, le manteau de Dieu, ce qui suppose qu'il a un corps ${ }^{63}$. Seule la compilation de Máase Merkaba ne dit absolument rien sur l'apparence de Dieu $^{64}$. Les textes de la merkaba manifestent souvent une tension entre l'aspiration du mystique à voir Dieu et les dangers que comporte la réalisation de cette aspiration ${ }^{65}$.

Le corpus mystique donne une grande importance au nom de Dieu et, à plusieurs reprises, établit un lien entre le corps de Dieu et son nom ${ }^{66}$. Dieu peut donc être décrit de manière corporelle, soit sous la forme d'un corps anthropomorphe, soit sous la forme d'un corps linguistique, constitué de lettres. Cette conception double de la corporéité divine est très proche de ce que Moshe Idel appelle le morphonominalisme ${ }^{67}$. La ressemblance entre Dieu et son fils se traduit sur deux plans différents, mais souvent articulés l'un avec l'autre : un plan extérieur (exprimé par les termes de "visage ", « image », " sceau », « luminosité ", 
« beauté » et " fils ») et un plan intérieur (avec les termes " souffle », « esprit », « parole », « nom »). À première vue, le nom est plutôt considéré par M. Idel comme une dimension spirituelle, distincte du corps, mais la situation est en réalité plus complexe. Le terme de morphonominalisme suppose une étroite affinité entre la forme et le nom, ce qui invite à penser que le nom a lui aussi une dimension corporelle. M. Idel parle d'ailleurs à plusieurs reprises d'iconisme linguistique ${ }^{68}$. L'origine $\mathrm{du}$ morphonominalisme remonte vraisemblablement au commentaire d'Ex 23, 20-24 et de la figure angélique que ces versets décrivent. On le retrouve dans la littérature apocalyptique, qumranienne et gnostique (valentinienne) ainsi que dans la littérature talmudique et celle des Hekhalot. Il est un trait récurrent de la conception juive de la filiation mystique, aux époques médiévale et moderne ${ }^{69}$.

La double révélation du corps de Dieu (l'Anthropos dont on décrit les mensurations et le nom), caractérise les traditions du Shiur Qoma et la gnose de Marc ${ }^{70}$. Sans parler de morphonominalisme, comme le fait M. Idel, Moses Gaster ${ }^{71}$ et Gershom Scholem ${ }^{72}$ avaient déjà perçu la parenté de ces deux corpus. Le lien entre le corps divin et le nom est enfin présent dans les sources judéo-chrétiennes ${ }^{73}$.

\section{Débats sur les sources rabbiniques}

Les textes que nous avons cités ont suscité un grand nombre de débats ${ }^{74}$. Pour le corpus mystique, on s'est demandé dans quelle mesure il est représentatif de la pensée des rabbins. Ne contient-il pas des conceptions très isolées, voire hétérodoxes? Les travaux de G. Scholem ou encore de Saül Lieberman insistent au contraire sur l'ancienneté d'une part significative du corpus mystique et sa concordance avec certaines traditions rabbiniques. Une telle concordance existe par exemple sur l'interprétation du Cantique des Cantiques comme décrivant la vision de Dieu par Israël au Sinaï, sur le thème du manteau ou du nom comme manifestation corporelle de Dieu ${ }^{75}$. L'idée d'un Dieu aux mensurations gigantesques peut être rapprochée des traditions aggadiques, selon lesquelles la taille d'Adam allait de la terre au ciel ou d'une extrémité du monde à l'autre ${ }^{76}$ . Le problème fondamental reste cependant celui de la lecture des textes, que nous avons déjà rencontré dans le cadre de la Bible. Faut-il tous les lire de manière littérale ou est-il possible de donner à certains d'entre eux une signification métaphorique ou allégorique? Certains textes résistent d'emblée à la lecture allégorique. C'est le cas, par exemple, de l'interprétation par Rabbi Eliezer du verset d'Ex 15, 2 : «Celui-ci (ze) est mon Dieu ». C'est l'emploi du démonstratif ze qui lui fait dire qu'une servante «a vu au bord de la Mer (Rouge) ce que n'a pas vu Isaïe ainsi qu'Ezéchiel». Les Hébreux ont bien vu Dieu concrètement, au point de pouvoir le pointer du doigt! Reste à savoir si tous les textes manifestent une résistance semblable. Depuis l'époque médiévale, la tendance lourde de l'herméneutique juive est d'interpréter ces textes midrashiques dans un sens allégorique. Maïmonide en est l'exemple le plus célèbre. Cette tendance lourde est également bien représentée dans la littérature universitaire. Alexander Altmann semble considérer que la théologie des rabbins est fondamentalement hostile à l'anthropomorphisme ${ }^{77}$. Cette conviction est également partagée par d'autres commentateurs ${ }^{78}$. Le corporéisme des rabbins ne serait que partiel selon Morton Smith et A. Marmorstein ${ }^{79}$.

David H. Aaron s'est livré à une reprise minutieuse de l'article d'A. Goshen Gottstein ${ }^{80}$. Il estime que ce dernier a pris certains textes à la lettre, alors qu'ils peuvent être lus de manière métaphorique. C'est le cas, par exemple, du texte sur le talon d'Adam, où 
l'expression imagée qelasțor panaw signifie la beauté du visage et n'implique nullement que le visage est une source réelle de lumière. Le texte est par ailleurs tissé en profondeur par des rapprochements sémantiques : la pomme, la sphère du soleil ou les disques dont parle la comparaison qui se trouve dans la suite du texte, font tous allusion à l'idée de rondeur. La cohérence du texte est donc bien plus située au niveau d'un certain nombre d'effets littéraires que dans l'éventuel référent d'un Adam lumineux ${ }^{81}$.

La littérature mystique, par définition, attire plus encore le soupçon «allégorique ». Les mystiques n'ont-ils pas une tendance bien connue à utiliser des images pour cacher leurs enseignements ou pour tenter d'exprimer ce qui, par définition, échappe à l'entendement ordinaire ? Comme le note $\mathrm{G}$. Stroumsa, la mystique chrétienne a cultivé dans un premier temps le corporéisme, avant de se tourner vers une vision beaucoup moins concrète de la divinité, en termes de lumière ${ }^{82}$. Cela invite à se demander si la lumière n'aurait pas aussi, chez les rabbins de l'Antiquité, un caractère métaphorique. Les textes de la merkaba promettent souvent une description physique de la divinité pour finalement nous révéler... ses noms. On peut comprendre par là, comme nous l'avons fait plus haut, que le nom est aussi une manifestation corporelle de la divinité. On peut aussi en déduire que Dieu n'a pas de forme physique, si bien que le mystique doit « se contenter » de ses noms 83. Peter Schäfer semble pencher pour cette dernière lecture ${ }^{84}$. Même le Sh'iur Qoma n'échappe pas à l'herméneutique du soupçon. Il est peut-être, par ses outrances, le mieux placé pour se prêter à l'interprétation allégorique. La démesure de ses indications chiffrées exprimerait l'infinité de Dieu et le caractère insaisissable de son essence ${ }^{85}$.

En dépit de toutes ces considérations, la tendance dominante de la recherche actuelle est de lire les textes de manière littérale et d'admettre que les rabbins de l'Antiquité croyaient bel et bien dans un Dieu corporel. Il est d'abord difficile d'extraire un texte de son sens littéral si aucun indice vraiment convaincant ne pousse en ce sens. C'est même le principe fondamental qui sous-tend toute l'exégèse midrashique des rabbins de l'Antiquité : un verset doit présenter un certain nombre d'anomalies ou d'indices formels qui rendent son sens littéral insatisfaisant, pour recevoir une autre lecture, de type midrashique ${ }^{86}$. Quand ces anomalies ou ces indices sont absents, le midrash n'hésite pas à en rester à la lettre du verset. C'est le cas, par exemple, pour Dt 34, $5:$ «Et Moïse mourut par la bouche de l'Éternel ('al pi ha-shem). » Comme rien ne permet, dans le verset, de douter qu'il s'agisse bien d'une bouche, le midrash reste fidèle à la lettre et affirme que Moïse est mort par un baiser de Dieu ${ }^{87}$. L'interprétation allégorique manque par ailleurs d'un fondement vraiment solide. Aucun texte n'affirme explicitement que Dieu est dénué de corps et la lecture allégorique n'a pas vraiment de statut codifié dans la littérature du midrash. Dans plusieurs textes, la corporéité de Dieu sert à fonder des pratiques concrètes, ce qui n'est guère concevable si elle se réduit à une simple allégorie ${ }^{88}$. La cohérence du corpus, en dépit des différences d'opinion fréquentes chez les rabbins, est également un fait à prendre en compte. Si un même motif est utilisé de manière apparemment littérale dans un grand nombre de textes, il est difficile de lui donner partout un sens métaphorique, parce que dans l'une de ses occurrences, certains indices justifient cette lecture. Il faut aussi tenir compte du mode de pensée très particulier des rabbins. Comme le note Yair Lorberbaum, et c'est sa thèse centrale, interpréter les textes corporéistes de manière allégorique est un contresens complet, car ils relèvent d'un mode de pensée mythique ou théurgique. Shamma Friedman abonde dans le même sens : il faut lire ces textes en fonction de leurs catégories conceptuelles propres et éviter autant que possible d'utiliser des notions étrangères à l'univers mental de leurs auteurs ${ }^{89}$. Il est 
probable cependant que la réaction corporéiste des chercheurs a été trop forte : il est toujours délicat, comme le fait, par exemple, A. Goshen Gottstein, de réduire un corpus aussi pluriel que celui des rabbins à une seule opinion. La conception corporelle de Dieu est dominante mais certainement pas unique, surtout si l'on prend en compte la littérature targumique et sa tendance à réduire anthropomorphismes et anthropopathismes ${ }^{90}$. Des travaux récents ont contribué à relativiser cette tendance, sans la nier entièrement pour autant ${ }^{91}$.

Le texte suivant, tiré du Talmud Babli, est également une sérieuse objection à ceux qui affirment que les rabbins de l'Antiquité ne connaissent pas la notion d'un Dieu incorporel : «Le Saint, béni soit-il, voit et n'est pas vu/n'est pas visible, de même l'âme voit et n'est pas vue/n'est pas visible ${ }^{92}$. » Le texte compare Dieu et l'âme et il insiste sur le fait que l'un comme l'autre ne sont pas visibles: ces deux éléments vont dans le sens de l'incorporéité divine. On pourrait objecter que l'âme n'est pas nécessairement une entité incorporelle pour les rabbins ${ }^{93}$. On peut aussi suivre l'autre traduction possible: « et (Dieu) n'est pas vu». En d'autres termes, la plupart du temps, Dieu n'est pas vu, mais il reste susceptible d'être vu par quelques privilégiés et dans certaines circonstances particulières.

31 Les partisans de la lecture allégorique pourraient tirer partie d'un groupe particulier de textes, celui qui traite de la polymorphie divine. Le midrash suivant en est un bon exemple :

«"Je suis l'Éternel, ton Dieu" (Ex 20,2), parce que le Saint, béni soit-Il, leur est apparu dans la Mer comme un guerrier qui fait la guerre (allusion à Ex 15, 3), il ${ }^{94}$ s'est révélé à eux au Sinaï, comme un scribe qui enseigne la Tora et il leur est apparu à l'époque de Daniel comme un ancien qui enseigne la Tora (allusion à Dn 7 , 9), il leur est apparu, à l'époque de Salomon, (comme) un jeune homme (Ct 5, 15). Le Saint, béni soit-Il, leur a dit: Ce n'est pas que vous méritiez de me (voir) sous des formes diverses (demuyot harbe), mais c'est moi qui étais dans la Mer, c'est moi qui étais au Sinaï, "je suis l'Éternel, ton Dieu" (Ex 20, 2) ${ }^{95}$."

Si Dieu change de forme à volonté, c'est qu'aucune de ces formes n'est vraiment la sienne et que dans le fond il n'a pas de corps propre et donc pas de corps ${ }^{96}$. Cette objection, à première vue pertinente, invite à se poser la question suivante : quel est le statut exact du corps divin dont la littérature rabbinique nous parle?

\section{Le statut du corps divin}

33 Le corps divin est-il celui de la divinité elle-même ou uniquement une manifestation corporelle qui serait distincte de son essence, une sorte d'hypostase ? À première vue, ces distinctions paraissent très abstraites, mais elles permettent de mieux comprendre la nature d'un personnage très particulier de la littérature rabbinique, l'ange Métatron, dont le nom "est comme le nom de son maître ${ }^{97}$. L'identité du nom, surtout quand il s'agit du Tétragramme, n'est jamais une affaire simplement linguistique. Métatron serait la manifestation corporelle de Dieu, une hypostase archangélique, pour reprendre l'expression de G. Stroumsa ${ }^{98}$. Ainsi défini, il peut aisément être rapproché de Jésus avec lequel il partage plusieurs points communs. Métatron comme Jésus ont, par exemple, deux noms : un nom exotérique de six lettres et un nom caché de vingt-quatre lettres ${ }^{99}$. Ils possèdent aussi la capacité de changer de forme et se présentent tantôt sous l'apparence d'un jeune homme, tantôt sous celle d'un vieillard ${ }^{100}$. M. Idel apporte également une pièce au dossier, en affirmant que Métatron est, à bien des égards, un véritable fils de Dieu ${ }^{101}$. La 
notion d'hypostase archangélique existait déjà dans le judaïsme du $\mathrm{I}^{\mathrm{er}}$ siècle et elle a vraisemblablement rendu possible la doctrine chrétienne de l'incarnation ${ }^{102}$. Il est possible que la littérature rabbinique évoque d'autres hypostases de la divinité que Métatron, celle de l'Adam cosmique ${ }^{103}$ ou encore celle de la Shekhina ${ }^{104}$. Le statut de la Shekhina n'est pas clairement défini par les rabbins de l'Antiquité et ceux du Moyen Âge sont très divisés sur la question: Sa'adya Ga'on et Maïmonide affirment qu'elle est une créature de Dieu alors que pour Nahmanide, la Shekhina est Dieu lui-même ${ }^{105}$. Certaines traditions laissent penser que la distinction intradivin/extradivin est trop tranchée et que la nature de la Shekhina est plus complexe : elle serait une hypostase de la divinité, par laquelle celle-ci se manifeste de manière sensible, sous forme de lumière ${ }^{106}$.

L'idée que Dieu a un corps s'impose donc pour les rabbins de l'Antiquité, même si la nature exacte de ce corps peut être objet de débat ${ }^{107}$. Cette idée, une fois prise en compte, doit amener à repenser en profondeur les relations du judaïsme rabbinique avec le paganisme ainsi qu'avec le judéo-christianisme et le christianisme.

\section{Judaïsme et paganisme}

On a coutume d'opposer le paganisme avec ses dieux visibles et anthropomorphes et le judaïsme avec son dieu invisible et transcendant. Cette opposition est, pour commencer, une simplification excessive des religions païennes. Leurs divinités peuvent être également zoomorphes ${ }^{108}$. Elles peuvent être aussi l'objet d'un culte aniconique : c'est le cas par exemple dans la religion perse des Sassanides. Richard Kalmin a montré que la perception de l'idolâtrie par les rabbins babyloniens a évolué au fil du temps, le paganisme des Parthes, à base d'images, laissant place à celui, aniconique, des Sassanides 109. La raréfaction des idoles semble entraîner chez eux une anxiété croissante à leur égard ${ }^{110}$. Quant au judaïsme rabbinique, on l'a vu, il croit en un Dieu corporel, même si on ne peut le représenter de manière plastique, sa représentation matérielle par excellence étant l'homme vivant. Les païens comme les rabbins partagent donc la croyance en un dieu corporel. L'accusation de paganisme que certains Pères brandissent à l'égard du judaïsme est donc loin d'être uniquement une accusation polémique ${ }^{111}$. Elle découle aussi d'une convergence objective du judaïsme et du paganisme sur la question du corps divin. $\mathrm{Si}$ cette convergence a été perçue par certains Pères de l'Église, il est peu probable qu'elle ait échappé aux rabbins eux-mêmes. L'emploi par la Bible des mêmes termes (șelem, temuna) pour désigner l'être humain en tant qu'il ressemble à Dieu et l'idole est un fait qui leur était certainement connu.

Certains textes rabbiniques mettent explicitement en parallèle l'idole et la représentation de Dieu qu'est l'homme. Le parallèle insiste parfois sur la ressemblance ou l'analogie entre ces deux éléments. C'est le cas de l'histoire de Hillel, citée plus haut, qui compare la statue du roi («les statues des rois»), représentation d'une divinité pour les païens, à l'homme (« moi qui suis créé à l'image et à la ressemblance »), représentation de Dieu, sur le plan corporel ${ }^{112}$. Un autre texte déjà cité compare la destruction des images et des statues des rois avec une diminution de la ressemblance de Dieu, c'est-à-dire la destruction d'un corps humain par le meurtre ${ }^{113}$. Les rabbins n'hésitent pas à faire dire à Jacob par les anges : " "Israël, avec lequel je m'orne" (Is 49,3$)$. Tu es celui dont l'image (iqonin) est gravée en haut ${ }^{114}$. » Selon S. Friedman, gravé « en haut » signifie "sur le visage même de Dieu ». Les versions ultérieures de cette aggada, qui parlent d'une image gravée sur le trône de gloire, en affaiblissent la portée, vraisemblablement polémique à l'égard du 
statut attribué à Jésus en Jn $1,51-52^{115}$. Le texte exprime surtout un curieux renversement : c'est Dieu qui est la représentation plastique de Jacob et dans le parallèle avec le roi et sa statue, c'est Jacob qui correspond au roi et Dieu à la statue. Le terme iqonin, employé ailleurs dans le sens de "statue » du roi, désigne, dans notre texte, la reproduction des traits de Jacob sur le visage de Dieu.

En dépit de l'interdiction de représenter des visages humains sur les pièces, les rabbins mentionnent quatre exceptions, Abraham, Josué, David et Mardochée ${ }^{116}$. La tradition suivante compare les statues du roi avec le corps divin, cette fois-ci représenté par son nom :

" Autre interprétation de "Louez le nom de l'Éternel" (Ps 113, 1). Parabole. À quoi la chose est-elle comparable? À un roi qui envoya son escorte à la guerre et qui lui donna sa/ses statues (iqonin). Il lui dit: À chaque fois que tu remporteras des victoires, tu loueras mes statues et ce sera comme si tu me louais moi. C'est pourquoi il est dit : "Louez le nom de l'Éternel ${ }^{117}$." »

Certaines traditions, plus économiques, comparent directement Dieu à l'idole, sans passer par la médiation de l'homme. Dieu est, par exemple, comparé à une statue avec un visage de chaque côté, ce qui revient à l'identifier avec Janus Quadrifrons ${ }^{118}$.

D'autres parallèles entre l'homme, image (corporelle) de Dieu et la statue, image du dieu païen, insistent au contraire sur ce qui les distingue. C'est le cas dans les Homélies Pseudoclémentines: "Vous êtes l'image du Dieu invisible. Que ceux donc qui désirent vivre pieusement ne disent pas que les idoles sont des images de Dieu [...] car l'image de Dieu, c'est l'homme [...] parce que le corps de l'homme porte l'image de Dieu ${ }^{119}$. » L'idole est une fausse image de la divinité, le corps de l'homme en est une vraie. En dépit de cet aspect critique, les Homélies Pseudo-clémentines fournissent certainement le parallèle le plus explicite des deux types d'images que sont l'idole et le corps humain, comme elles avaient été les plus claires à affirmer le caractère lumineux du corps divin.

Le Midrash donne enfin l'exemple d'un parallèle qui articule la ressemblance et la différence: "“Elles ont des oreilles et n'entendent pas" (Ps 115, 6). Que deviennent sourdes les oreilles qui entendent (de ceux) qui adorent les oreilles qui n'entendent pas et qui délaissent les oreilles qui entendent, ainsi qu'il est dit : "L'Éternel écouta et entendit" $(\mathrm{Ml} \mathrm{3,16})^{120}$. » Les idoles comme le Dieu unique ont des yeux (ressemblance), mais les yeux des idoles ne voient pas alors que ceux de Dieu voient (différence).

Quelques traditions ne se contentent pas de tracer un parallèle entre la statue et le corps de Dieu mais vont jusqu'à établir un lien causal entre les deux. Ainsi il est interdit de représenter Dieu (comme une idole) non parce qu'il est dénué de corps mais parce que son corps n'a rien de comparable avec tous les autres corps existants :

« Et le jour du don de la Tora, le Saint, béni soit-il, a déchiré les cieux et a fait voir à Israël ce qu'il y a en haut. Rabbi Pinhas et Rabbi Lévi (ont dit) au nom de Rabbi Shim'on ben Laqish : Le Saint, béni soit-il, a déchiré pour eux (les) sept firmaments et de même qu'il a déchiré l'En-haut (ha-'elyonim), de même il a déchiré l'En-bas (hatahtonim), ainsi qu'il est dit: "qui est dans les cieux en haut et sur la terre en bas" (Ex 20,4). Voyez qu'il n'y en a pas d'autre avec moi, ainsi qu'il est dit : “C'est à toi qu'il a fait voir (tout cela) pour que (tu) saches (que l'Éternel est Dieu et qu'il n'y en a pas d'autre que lui)" (Dt 4, 35) (et) "Et sache aujourd'hui et fais revenir dans ton cœur que l'Éternel est Dieu, dans les cieux en haut et sur la terre en bas, il n'y en a pas d'autre" (Dt 4, 39) ${ }^{121}$. »

Il est également interdit de représenter l'homme parce que son corps est à l'image de celui de Dieu ${ }^{122}$. On pourrait objecter que si le corps de Dieu ressemble à celui de l'homme, 
il n'est plus aussi incomparable et atypique que l'affirme la première tradition citée. Cette divergence s'explique peut-être par deux conceptions du corps divin, une qui est anthropomorphe et une autre qui ne l'est pas. On peut aussi penser que la ressemblance corporelle entre Dieu et l'homme concernait surtout le premier homme, avant la faute. Le corps actuel de l'homme n'est plus qu'un reflet éloigné de celui de Dieu, ce reflet étant cependant suffisant pour justifier l'interdiction de représenter l'homme ${ }^{123}$. Mais la première tradition mentionne uniquement les créatures des cieux et des profondeurs, dont aucune n'est comparable à Dieu. Le texte ne cite pas explicitement le cas de l'homme. Les deux traditions qui nous occupent ne seraient donc pas contradictoires. Elles s'accordent sur un principe essentiel: c'est parce que Dieu a un corps qu'il est interdit de le représenter. Chacune traite ensuite un cas différent. La première aborde le cas des créatures dont le corps n'a rien de semblable à celui de Dieu. Représenter Dieu sous leurs traits serait donner une fausse image de la divinité. La deuxième tradition traite en revanche le cas particulier de l'homme : ce n'est pas la dissemblance entre Dieu et l'homme qui fait problème, mais au contraire leur ressemblance ${ }^{124}$. L'homme ne peut être représenté parce qu'il ressemble à Dieu et il est probable que la réciproque est vraie: Dieu ne peut être représenté parce qu'il ressemble à l'homme. La ressemblance entre le corps de Dieu et celui de l'homme comporte donc un risque majeur, celui d'effacer la différence fondamentale, aux yeux des rabbins, entre le créateur et la plus importante de ses créatures $^{125}$. Il reste que dans la première tradition, Dieu s'adresse directement à des hommes (les Hébreux), en leur disant: "Voyez qu'il n'y en a pas d'autre avec moi. » Comment peut-on affirmer que le cas de l'homme ne serait pas pris en compte ici? En fait, il est probable que Dieu a un objectif plus précis : montrer aux Hébreux que, dans les lieux cachés que sont les cieux et les profondeurs, il n'y a pas une entité qui aurait un corps identique à celui de Dieu et qui serait donc elle-même divine. Le texte a une orientation antignostique et il est donc parfaitement compatible avec l'idée que l'homme est la créature, dont le corps est le plus semblable à celui de Dieu, sans lui être identique.

Un lien causal peut également exister entre la statue et le corps des anges. Ainsi, les Hébreux, en faisant le veau d'or ont voulu reproduire la face de taureau des Hayyot qui portent le char divin (merkaba). Moïse sollicite pour cette raison la miséricorde divine. L'idole du veau d'or est en fait la reproduction directe d'un élément appartenant au corps d'un ange ${ }^{126}$.

Les rabbins établissent donc des parallèles entre la représentation corporelle de Dieu qu'est l'homme et celle de la divinité païenne qu'est l'image sculptée. Si l'on excepte le texte des Homélies Pseudo-clémentines, un peu périphérique par rapport au corpus des rabbins, l'ensemble des parallèles ne revêt pas un aspect négatif ou polémique. Est-on en présence d'un cas particulièrement incontestable d'hellénisation des rabbins? D'autres exemples renforcent l'idée que l'univers mental des rabbins est en partie perméable aux représentations païennes. En témoigne par exemple l'identification d'Isis avec Ève ou celle de Poséidon/Neptune avec le Prince de la $\mathrm{Mer}^{127}$. La présence d'Hélios dans les mosaïques des synagogues ou dans une prière du Sefer ha-razim n'est peut-être pas si éloignée que cela du judaïsme rabbinique antique ${ }^{128}$.

Le fait que des rabbins n'hésitent pas à faire des parallèles entre l'homme et l'image sculptée, dans un contexte autre que polémique, suppose en tout cas que l'image sculptée n'est pas considérée systématiquement de manière négative. Cette évolution, dans la perception des images, qui démarque le judaïsme rabbinique de celui qui l'a précédé, peut être observée dans un grand nombre de traditions ${ }^{129}$. Dans la Mishna, Rabbi Me'ir interdit 
toutes les images ou statues, car elles sont considérées par lui a priori comme des objets de culte. Les Sages, en revanche, n'interdisent que les statues qui ont dans la main un bâton, un oiseau ou un globe ${ }^{130}$. Yaron Eliav a bien montré que ces objets sont des attributs qui permettent de distinguer les statues des dieux de celles qui représentent de simples hommes ${ }^{131}$. Les Sages n'interdisent donc que les statues qui représentent explicitement des dieux. D'autres traditions contribuent encore à assouplir cette position. Une statue de déesse qui n'est pas objet de culte mais l'ornement d'un bain n'est pas problématique, comme le montre le dialogue entre Proclus, fils de Philosophos et Rabban Gamliel, tous deux présents à Acre, "dans le bain d'Aphrodite ${ }^{132}$ ». Même si certains individus viennent honorer une statue de ce type, il ne s'agit pas d'un culte officiel et donc on ne le prend pas en compte ${ }^{133}$.

Certains rabbins sont également conscients que le paganisme n'est pas qu'un culte mais qu'il est aussi une croyance dans des divinités et celles-ci ne se confondent pas avec leurs statues ${ }^{134}$. Cette définition du paganisme comme croyance (ou absence de croyance) contribue encore plus à minorer l'importance de la question des images.

\section{Judaïsme et christianisme}

Si l'on se tourne vers le christianisme, la situation est plus complexe : l'existence d'un Dieu juif corporel contribue-t-elle à rapprocher (intellectuellement) le judaïsme du christianisme ou au contraire à les éloigner l'un de l'autre? Les études savantes sont assez divisées sur la réponse à apporter à cette question. Pour Daniel Boyarin, le judaïsme rabbinique et le christianisme ancien sont en radical désaccord sur la question du corps divin. Le judaïsme rabbinique part de l'idée que Dieu est immédiatement corporel. Le christianisme a au contraire une perspective dualiste : Dieu est d'abord invisible avant de s'incarner et de se rendre visible ${ }^{135}$. Ces deux conceptions différentes de la divinité dans son rapport au corps se retrouvent sur le plan de l'anthropologie et de l'herméneutique ${ }^{136}$ . Le judaïsme rabbinique conçoit l'homme comme un tout. Même quand il présente l'homme en termes dualistes, ce dualisme est peu accusé. La conception chrétienne du corps insiste au contraire sur la dualité des deux substances (âme et corps) et la tension qui existe entre elles. Dans le domaine de l'herméneutique, le christianisme ancien privilégie la lecture allégorique de l'Écriture. L'essentiel n'est pas la facette corporelle de l'Écriture, sa lettre, mais ce qu'il y a au-delà de la lettre, c'est-à-dire l'esprit, le sens spirituel du texte. On a souvent rapproché voire identifié l'approche allégorique chrétienne du midrash juif. Tous deux écartent en effet la lettre pour aller au-delà, vers ce qui n'est pas dit explicitement dans le texte et qui est le plus important. D. Boyarin insiste au contraire sur la différence profonde qui sépare l'allégorie chrétienne du midrash. Le midrash valorise la lettre, car c'est à partir des particularités de la lettre et de leur examen approfondi (talmud) que l'on accède au sens caché de l'Écriture. Le midrash n'est pas la quête d'une idée derrière la lettre du verset. Il est d'abord et surtout la tentative de revivre une expérience concrète, empirique, celle de la vision de Dieu ${ }^{137}$, tout particulièrement la vision sinaïtique. C'est pourquoi la lecture que font les rabbins du Cantique des Cantiques ne peut être qualifiée d'allégorique, car ce texte biblique est bien, pour eux, le récit d'une expérience concrète et physique, celle de la rencontre de Dieu avec Israël, à la Mer Rouge et au mont Sinaï. Ce désir de voir Dieu, qui s'exprime à travers la pratique du midrash et qui est même son essence, a un caractère érotique. Il n'est donc pas surprenant que les rabbins établissent un lien fort entre l'Exode et le Cantique des 
Cantiques $^{138}$. La conception qu'a D. Boyarin du lien entre midrash et corps divin présente certaines affinités avec les réflexions d'Elliot R. Wolfson sur la même question. Celui-ci s'appuie notamment sur la tradition qui loue la «faculté imaginative " (koah le-dammot) des prophètes ${ }^{139}$ : ces formes de Dieu, que le visionnaire (individu ou collectivité) perçoit, sont certes des constructions mentales mais elles ne sont pas que dans l'esprit du visionnaire, elles sont réelles. La faculté imaginative (koah le-dammot) est véritablement le medium de la rencontre avec Dieu ${ }^{140}$. Dans une étude récente,

E. R. Wolfson réaffirme ses positions antérieures. Le judaïsme rabbinique combine à la fois le rejet de l'incarnation chrétienne et une conception anthropomorphique de Dieu très affirmée et qui n'a pas une signification simplement métaphorique. En fait, les rabbins soutiennent que l'on peut avoir une expérience concrète de Dieu dans la prière et l'étude, celle du « corps imaginal de Dieu ». Le corps de Dieu est réel autant qu'il est imaginé et imaginé autant qu'il est réel. Le corps n'est pas qu'un objet, il est quelque chose de construit par l'imagination, de manière complexe ${ }^{141}$.

À l'inverse de D. Boyarin, G. Stroumsa estime que les conceptions anciennes, juives et chrétiennes, du corps divin sont très proches. La distinction entre un Dieu invisible et son hypostase visible n'est pas propre au christianisme, elle s'enracine dans des conceptions juives, déjà attestées au $\mathrm{I}^{\mathrm{er}}$ siècle. La double manifestation corporelle de cette hypostase, anthropomorphe ou nominale, est également commune au judaïsme et au christianisme, ce dernier étant là aussi directement influencé par son prédécesseur. La mystique originelle des chrétiens se distingue d'ailleurs mal de celle du judaïsme : elle présente la même orientation corporéiste. Par la suite, les descriptions physiques de Dieu tendent à disparaître des textes mystiques chrétiens. La place de la représentation plastique dans le christianisme ancien connaît une évolution inverse à celle de la représentation discursive, que nous venons de mentionner. Le christianisme, originellement aniconique, devient une religion iconique au $\mathrm{IV}^{\mathrm{e}}$ siècle de notre ère. Cette évolution n'a toujours pas fait l'objet d'une explication satisfaisante. G. Stroumsa propose de l'éclaircir à partir de la situation du judaïsme ancien. Celui-ci interdit la représentation plastique de Dieu. Il se montre en revanche beaucoup plus ouvert quand il s'agit de représentations discursives. N'y a-t-il pas un lien entre les deux phénomènes ? C'est l'interdit de la représentation plastique qui, par une sorte de compensation du refoulé, aurait stimulé le développement des visions anthropomorphiques de Dieu. Il est probable que la destruction du Second Temple, siège concret de la divinité, a contribué à stimuler plus encore l'imagination des rabbins et des mystiques. Loin d'être lus de manière allégorique par les rabbins, les anthropomorphismes de la Bible sont renforcés et l'on n'hésite pas, comme dans le Shiur Qoma, à décrire dans le détail les membres du corps de la divinité. Une explication semblable vaudrait pour le christianisme, mais dans le sens inverse. Plus la mystique chrétienne s'éloigne du corporéisme et plus le christianisme éprouve le besoin de s'appuyer sur des images concrètes et plastiques ${ }^{142}$.

Les positions de D. Boyarin et de G. Stroumsa sont donc difficiles à concilier, parce qu'elles ne concordent pas sur le statut exact du corps divin. Est-il le corps de la divinité elle-même, comme le pense D. Boyarin ou est-il uniquement une manifestation de celle-ci, sous forme d'hypostase, comme le soutient G. Stroumsa? Si la corporéité de Dieu s'impose comme la vision rabbinique majoritaire dans l'Antiquité, il est moins aisé de déterminer, à partir des textes, la nature précise de ce corps divin. La distinction entre l'essence de la divinité et sa manifestation, fortement conceptuelle voire philosophique, 
est loin d'être une évidence dans une littérature juive ancienne qui, Philon excepté, est peu férue, en apparence, du logos philosophique.

\section{NOTES}

1. Voir l'opinion représentative d'Origène dans Sur la prière, 23, 3, ainsi que l'étude de Gedaliahu Stroumsa, «L'incorporéité de Dieu : contexte et implications de la doctrine d'Origène ", in Savoir et salut, Paris, 1992, p.183-197. Deux exceptions sont à noter: la position de Tertullien, d'inspiration stoïcienne et celle des audiens et des moines du désert égyptien, comme le note G. Stroumsa dans " Forme(s) de Dieu : Métatron et le Christ ", in Savoir et salut, p. 66, n. 6.

2. Voir Aristote, Métaphysique, VII, 1072a-b ainsi que IX et Plotin, Ennéades, VI, VII, 37. Il n'est pas sûr que le démiurge de Platon soit un être entièrement spirituel (Timée, 29a-31b).

3. Le texte grec traduit est celui de Nestle-Aland, Novum Testamentum Graece, Stuttgart, 1993, p. 248. Certaines versions omettent la deuxième mention de Dieu et parlent $d u$ «fils unique » tout court. Sauf contre-indication expresse, tous les textes cités sont traduits par nos propres soins.

4. La situation est en fait plus complexe, puisque les Pères de l'Église soutiennent également, à la suite du premier chapitre de Jean, l'idée d'une préexistence du Fils. Voir sur ce point Harry Austryn Wolfson, «Extradeical and intradeical Interpretation of Platonic Ideas ", in Religious Philosophy. A Group of Essays, Cambridge, 1961, p. 27-68.

5. Voir Moshe Weinfeld, "The Uniqueness of the Decalogue ", in Ben-Zion Segal (éd.), The Ten Commandments As Reflected in Tradition (en hébreu), Jérusalem, 1985, p. 4-6 (et particulièrement la note 20).

6. Mishna, Rosh ha-shana, 2, 8, ms Kaufmann (Budapest) et Talmud Babli, Rosh ha-shana, 24a-b, ms de Munich. Conformément à la pratique de plus en plus répandue dans les études talmudiques actuelles, nous avons traduit les textes rabbiniques à partir de versions manuscrites, en l'occurrence celles disponibles sur le site de l'Académie de la langue hébraïque (http://hebrewtreasures.huji.ac.il).

7. Dialogue avec Tryphon, 114.

8. Homélies sur la Genèse, I, 13 et III, 1.

9. Sur l'origine de l'homme, I, 5 .

10. Contre les Gentils, III, 12.

11. G. Stroumsa, "Le couple de l'ange et de l'esprit. Traditions juives et chrétiennes ", in Savoir et salut, p. 30-33 et « Forme(s) de Dieu : Métatron et le Christ », id., p. 67-68.

12. Pour ces trois réponses, voir Daniel Boyarin, «The Eye in the Torah: Ocular Desire in Midrashic Hermeneutic ", in Sparks of the Logos. Essays in Rabbinic Hermeneutics, Leyde-Boston, 2003, p.3-4, dont les positions sont proches des nôtres. Pour la distinction entre la représentation plastique, directe ou indirecte et la représentation verbale, voir Moshe Halbertal et Avishai Margalit, Idolatry, Cambridge-Londres, 1992, p. 37-66.

13. Voir Mishna 'im perush Rabbenu Moshe ben Maymon. Maqor we-targum, éd. Yosef Kafih, t. IV, Seder Neziqin, Jérusalem, 1964, p. 211 ; Guide des Perplexes, II, 1-2 ; 'Eṣ Ḥayyim, heleq rishon, p. 14b et l'aperçu suggestif d'Henri Atlan, Les Étincelles de hasard, t. I. Connaissance spermatique, Paris, 1999, p. 89-96. Pour une vue d'ensemble de la conception maïmonidienne de l'incorporéité divine et de ses principaux enjeux, voir Shamma Friedman, "Șelem demut we-tabnit», Sidra, 22, 2007, 
p. 89-106 et pour une comparaison de la démarche maïmonidienne avec celle de Saint Augustin, voir, du même auteur, "Anthropomorphism and its eradication ", in Willem van Asselt, Paul van Geest, Daniela Müller, Theo Salemink (éd.), Iconoclasm and Iconoclash, Struggle for Religious Identity, Leyde, 2007, p. 158-168.

14. Celle-ci va même jusqu'à penser le judaïsme en termes d'incarnation, voir Moshe Idel, Ben : Sonship and Jewish Mysticism, Londres-New York, 2007, p. 62. La tendance à minorer la dimension corporelle de Dieu n'a cependant pas disparu de la recherche (M. Idel, op. cit., p. 112).

15. Sur la conception anthropomorphique et anthropopathique de Dieu dans la Bible, la littérature est vaste. Voir principalement James Barr, «Theophany and Anthropomorphism in the Old Testament », Vetus Testamentum, Supplément 7, Leyde, 1960, p. 31-38 ; Aaron Schart, « Die "Gestalt" YHWHs: ein Beitrag zur Körpermetaphorik alttestamentlicher Rede von Gott», Theologische Zeitschrift, 55, 1999, p. 26-43 et surtout Esther J. Hamori, «When Gods were men »: The embodied God in biblical and Near Eastern Literature, Berlin-New York, 2008.

16. E. J. Hamori, op. cit., p. 29-33.

17. Pour un choix de versets commentés, voir Guide des Perplexes, I, 28 (pied), 37 (face), 38 (dos) et 44 (œil).

18. Voir Gn 12,$7 ; 17,1 ; 18,1 ; 26,2 ; 26,24$ et 35, 9. Pour une liste plus complète, incluant d'autres personnages bibliques, voir Stephen D. Moore, "Gigantic God : Yahweh's body ", Journal for the Study of the Old Testament, 70, 1996, p. 95-96.

19. Sur l'existence d'un courant anti-anthropomorphique dans le Deutéronome, voir Moshe Weinfeld, Deuteronomy and the Deuteronomistic School, Oxford, 1972, p. 191-209. Sur le caractère problématique de la lecture anti-anthropomorphique du Deutéronome et du Deutéro-Isaïe, voir M. Halbertal et A. Margalit, Idolatry, p. 46 et S. Friedman, "Ṣelem demut we-tabnit ", Sidra, p. 107-109.

20. Sur cette ambivalence, voir S. D. Moore, "Gigantic God: Yahweh's body ", p. 93 et Elliot R. Wolfson, Through a Speculum that Shines: Vision and Imagination in Medieval Jewish Mysticism, Princeton, 1994, p. 21.

21. Voir Nb 33, 52 ; 1 S 6, 5 ; 6, 11 ; 2 R 11, 18 ; Ez 7, $20 ; 16,17$; 23, 14 ; Am 5, 26 ; 2 Cr 23, 17. Le mot șelem signifie « idole » dans la majorité des versets où il apparaît.

22. Il faut nécessairement rester prudent dans l'emploi de ce terme, car l'anthropologie biblique n'est pas dualiste. C'est même un argument de poids pour ne pas écarter la composante corporelle de l'être humain, dans l'interprétation du mot șelem (S. D. Moore, op. cit., p. 95).

23. Voir le commentaire de Sa'adya Ga'on sur Gn 1, 26-27 (Perushe Rabbenu Sa'adya Ga'on 'al ha-tora , éd. Yosef Kafih, Jérusalem, 1963, p. 12-13). Cette lecture est cependant totalement absente du judaïsme rabbinique ancien, comme le souligne Alon Goshen Gottstein (" The Body as Image of God in Rabbinic Literature ", Harvard Theological Review, 87, 1994, p. 185). Dans le corpus chrétien, elle remonte au moins à Chrysostome et elle concorde bien avec certaines représentations égyptiennes et mésopotamiennes (S. D. Moore, « Gigantic God : Yahweh's body », p. 93, n. 18).

24. Voir S. D. Moore, op. cit., p. 93-94.

25. Philon estime que la notion d'incorporéité est exprimée dans la Bible par celle de « dissemblance » : rien n'est comme Dieu ou semblable à lui (Harry Austryn Wolfson, Philo, t. II, Cambridge, 1962, p. 96).

26. Voir Guide des Perplexes, II, 25.

27. La lecture allégorique et rationaliste d'un certain nombre de versets est l'une des causes des deux controverses maïmonidiennes de 1230-1233 et 1303-1306, comme l'a montré Charles Touati (« La controverse de 1303-1306 autour des études philosophiques et scientifiques », in Prophètes, talmudistes et philosophes, Paris, 1990, p. 203-205).

28. Le cas de Spinoza est assez emblématique (Traité théologico-politique, VII).

29. Arthur Marmorstein, The Old Rabbinic Doctrine of God, II. Essays in Anthropomorphism, New York, 1937. 
30. A. Marmorstein, op. cit., p. 50-56.

31. A. Goshen Gottstein, "The Body as Image of God», p. 172. Sur la nécessité de distinguer anthropomorphisme et anthropopathisme et sur les erreurs d'interprétation d'A. Marmorstein, voir également S. Friedman, « Șelem demut we-tabnit », Sidra, p. 91 et 111-112.

32. Voir Bereshit Rabba, 8, 3 et 4 et Talmud Babli, Sanhedrin, 38b.

33. A. Goshen Gottstein, « The Body as Image of God», p. 189, n. 57.

34. Wa-yiqra Rabba, 34, 3, ms de Londres $340: 2$.

35. Talmud Babli, 'Aboda Zara, 43b, ms de Paris 1337.

36. Mekhilta de-Rabbi Yishma'el, Yitro, Ba-hodesh, 8, ms d'oxford 151.

37. Abot de-rabbi Natan, A, 2. Nous avons traduit la version de l'editio princeps de Venise, telle qu'elle est transcrite dans Avot de-Rabbi Natan, Synoptische Edition beider Versionen, éd. par HansJürgen Becker (en collaboration avec Christoph Brenner), Tübingen, 2006, p. 46. Voir aussi la version du ms de Vatican 44, Synoptische Edition, p. 296.

38. Yaron Eliav, "Viewing the Scriptural Environment : Shaping the Second Commandement ", in Peter Schäfer (éd.), The Talmud Yerushalmi and Graeco-Roman Culture, t. III, Tübingen, 2002, p. 416.

39. Sur la notion de gloire dans la Bible et les sources rabbiniques, voir E. R. Wolfson, Through a Speculum that Shines, p. 22 et 45-48.

40. Voir Sifre Debarim, § 355, Midrash Mishle, 10, éd. Buber, p. 34a et les ajouts de la Pesiqta Rabbati, 1, éd. Friedmann, p. 194a.

41. Voir, par exemple, l'interprétation d'Ez 43, 2 dans Abot de-rabbi Natan, A, 11.

42. Voir Ephraïm Urbach, Les Sages d'Israël. Conceptions et croyances des maîtres du Talmud, Paris, 1996, p. 47-49.

43. Talmud Babli, Berakhot, 17a, manuscrit d'Oxford 366 .

44. Bereshit Rabba, 3, 4, ms de Vatican 60. Voir les études de Victor Aptowitzer, «Zur Kosmologie der Aggada. Licht als Urstoff », Monatsschrift für Geschichte und Wissenschaft des Judentums, 1928, p. 363-370 et Alexander Altmann, "A Note on the Rabbinic Doctrine of Creation ", Journal for Jewish Studies, 7, 1956, p. 195-206.

45. A. Goshen Gottstein, «The Body as Image of God », p. 178 et ss.

46. Wa-yiqra Rabba, 20, 2, ms de Londres $340: 2$.

47. Bereshit Rabba, 20, 12, ms de Vatican 60.

48. Homélies Pseudo-clémentines, 17, 7 (trad. André Siouville, p. 322).

49. A. Goshen Gottstein, « The Body as Image of God », p. 172-173.

50. Sur le rapport entre l'ésotérisme et le christianisme primitif, voir Gedaliahu Stroumsa, Hidden Wisdom : Esoteric Traditions and the Roots of Christian Mysticism, Leyde-New York-Cologne, 1996.

51. Voir Sifre Debarim, $\S 355$, qui est au centre de l'étude de Michael Fishbane, « The Measures of God's Glory in the Ancient Midrash », in Ithamar Gruenwald, Shaul Shaked, Gedaliahu Stroumsa (éd.), Messiah and Christos: Studies in the Jewish Origins of Christianity Presented to David Flusser in the Occasion of this Seventy-Fifth Birthday, Tübingen, 1992, p. 53-74. À rapprocher aussi de Talmud Babli, Sukka, 45b : Moïse a vu Dieu à travers un «miroir qui brille» (transparent) et les autres prophètes à travers un « miroir qui ne brille pas » (teinté).

52. Voir Mekhilta de-Rabbi Yishma'el, Be-shallah, Ha-shira, 3 et Yitro, Ba-hodesh, 2 ainsi que l'ensemble de textes, plutôt tardifs, cités par M. Fishbane à la fin de son étude, op. cit., p. 73-74.

53. Midrash Tanhuma, Șaw, 13. Voir également Sifre Ba-Midbar, § 103.

54. Sifre Debarim, § 357.

55. Par exemple, entre Ex 24, 10-11 et 33, 20 ou entre Ex 19, $11 ; 24,10-11$ et Dt 4, 11.

56. Mekhilta de-Rabbi Yishma'el, Be-shallah, Ha-shira, 3, ms d'Oxford 151.

57. Le lien explicite entre Ex 15, 2 et « montrer du doigt » est fait dans Shir ha-shirim Rabba, 2, 14, 3.

58. Mekhilta de-Rabbi Yishma'el, Yitro, Ba-hodesh, 2, ms d'Oxford 151. 
59. Voir Martin Cohen, The Shi'ur Qoma: Texts and Recensions, Tübingen, 1985.

60. Saul Lieberman, Mishnat Shir ha-Shirim, in Gershom Scholem, Jewish Gnosticism, Merkabah Mysticism and Talmudic Tradition, New York, 1960, p. 123. Martin Cohen rejette ce point de vue dans The Shi ur Qoma : Liturgy and Theurgy in Pre-Kabbalistic Jewish Mysticism, Lanham, 1983.

61. Ces textes sont cités dans Merkaba Rabba (Peter Schäfer, Synopse zur Hekhalot Literatur, Tübingen, 1981, § 688-692, 694, 696-699, 704; id., Le Dieu caché et révélé, Introduction à la mystique juive ancienne, Paris, 1993, p. 101-104).

62. Les Hekhalot Rabbati donnent très peu d'indications sur l'apparence concrète de Dieu (Synopse, $\S 102,159$ et 198, Le Dieu caché et révélé, p. 21-26). Les Hekhalot Zuțarti sont plus loquaces sur la question (Synopse, § 352 et 356, Le Dieu caché et révélé, p. 61-64).

63. Hekhalot Rabbati, Synopse de P.Schäfer, $\S 102$ où il est question du vêtement (haluq) de « ZHRRY'L, le Seigneur, le Dieu d'Israël ».

64. P. Schäfer, Le Dieu caché et révélé, p. 81-84 (si l'on excepte l'identification de Dieu et du feu tirée de Dt 4, 24). La compilation de III Hénoch accorde une place essentielle à la notion de Shekhina, mais sans lui donner une dimension corporelle très marquée: "quant à la manifestation concrète de Dieu, elle est reléguée au second plan... » (op. cit., p. 142).

65. Voir P. Schäfer, Le Dieu caché et révélé, p. 25.

66. Synopse, § 102, 350, 551-552, 549, 689, 692, 694-704 et P. Schäfer, Le Dieu caché et révélé, p. 62 et 100.

67. M. Idel, Ben : Sonship and Jewish Mysticism, p. 18-19.

68. M. Idel, op. cit., p. 222 et 293.

69. M. Idel, op. cit., p. 20-22, 27-28, 114-123, 222, 226, 293 et 380.

70. Irénée, Contre les Hérésies (éd. Harvey, I, p. 128-134).

71. Moses Gaster, « Das Schiur Komah », in Studies and Texts, II, Londres, 1923-1928, p. 1330-1353.

72. Gershom Scholem, La mystique juive. Les thèmes fondamentaux, Paris, 1985, p. 46-49.

73. Voir Gedaliahu Stroumsa, «Un Dieu sans nom. "Théologies du Nom" judéo-chrétiennes et gnostiques », in Le Rire du Christ. Essais sur le christianisme antique, Paris, 2006, p. 55-62.

74. On trouve un bon aperçu des débats dans l'ouvrage de Yair Lorberbaum, Șelem Elohim: Halakha we-aggada, Tel Aviv, 2004, p. 27-82 et dans E. R. Wolfson, Through a Speculum that Shines, p. 23.

75. Voir G. Scholem, La mystique juive, p. 37-72 et Jewish Gnosticism, p. 58-64 ; S. Lieberman, Mishnat Shir ha-Shirim, p. 118-126. Voir aussi Midrash Hallel, in Bet ha-Midrash (éd. Adolf Jellinek, 6 vol., Vienne, 1853-1878), t. V, p. 88 qui compare le nom de Dieu et l'image ou la statue d'un roi.

76. Talmud Babli, Hagiga, 12a. Sur le rapprochement entre les corps gigantesques de Dieu et d'Adam, voir A. Goshen Gottstein, «The Body as Image of God», p.191-193 et S. D. Moore, "Gigantic God: Yahweh's body», p.100. Les deux attributs du corps divin (luminosité, gigantisme), sur lesquels insiste A. Goshen Gottstein, sont bien ceux qu'un grand nombre de juifs, contemporains de Maïmonide, attribuaient à Dieu (Guide des Perplexes, I, 1).

77. Alexander Altmann, "Homo Imago Dei" in Jewish and Christian Theology ", Journal of Religion, 48, 1968, p. 235-259.

78. Voir Y.Lorberbaum, Selem Elohim, p. 17, 33 et 44. Contrairement à Joshua Abelson (The Immanence of God in Rabbinical Literature, Londres, 1912, p. 82-97), E. Urbach refuse l'identification de la Shekhina avec une lumière matérielle (Les Sages d'Israël, p. 47 et 50-53).

79. A. Marmorstein, The Old Rabbinic Doctrine of God, p. 1-157 ; Morton Smith, « On the Shape of God and the Humanity of Gentiles ", in Jacob Neusner (éd.), Religions in Antiquity, Leyde, 1970, p. 315-326.

80. David H. Aaron, «Shedding Light on God's Body in Rabbinic Midrashim : Reflections on the Theory of a Luminous Adam », Harvard Theological Review, 90, 1997, p. 299-314.

81. D. H. Aaron, « Shedding Light on God's Body », p. 303-305. 
82. Gedaliahu Stroumsa, "To See or not to See: On the Early History of the Visio Beatifica ", in Peter Schäfer (avec la collaboration d'Elisabeth Müller-Luckner) (éd.), Wege mystischer Gotteserfahrung. Judentum, Christentum und Islam, Munich, 2006, p. 69.

83. Voir le $§ 699$ de la Synopse: nous ne possédons aucune mesure mais les noms nous sont révélés.

84. P. Schäfer, Le Dieu caché et révélé, p. 100.

85. Pour une lecture non littérale du Sh'iur Qoma, voir Joseph Dan, « The Concept of Knowledge in the Shi'ur Qoma ", in Siegfried Stein, Raphael Loew (éd.), Studies in Jewish Religious and Intellectual History Presented to A. Altmann, University of Alabama, 1979, p. 67-73.

86. Voir José Costa, La Bible racontée par le Midrash, Paris, 2004, p. 6-7.

87. La lecture littérale du terme «bouche » dans Dt 34, 5 est très répandue sans être cependant unique : voir sur ce point, Peter Schäfer, Klaus Haacker, « Nachbiblische Traditionen vom Tod des Moses », in Otto Betz, Klaus Haacker et Martin Hengel (éd.), Josephus Studien. Untersuchungen zu Josephus, dem antiken Judentum und dem Neuen Testament, Festschrift für O. Michel, Göttingen, 1974, p. $166-170$.

88. Voir S. Friedman, « Șelem demut we-tabnit », Sidra,p. 115.

89. S. Friedman, op. cit., p. 91 et 94.

90. Pour des raisons de place, il ne nous a pas été possible d'intégrer les textes du Targum dans notre développement. Voir cependant: Moses Ginsburger, Die Anthropomorphismen in den Targumim, Braunschweig, 1891.

91. Voir Michael L. Klein, Anthropomorphisms and Anthropopathisms in the Targumim of the Pentateuch (en hébreu), Jérusalem, 1982.

92. Talmud Babli, Berakhot, 10a, ms d'Oxford 366.

93. Sur la "matérialité » éventuelle de l'âme, voir les textes cités dans José Costa, L'au-delà et la résurrection dans la littérature rabbinique ancienne, Paris-Louvain, 2004, p. 480-487 et p. 482, n. 18.

94. Dans le ms d'Oxford 151, utilisé ici, on lit en fait : « comme il s'est révélé à eux au Sinaï ».

95. Pesiqta de-rab Kahana, 12, 24, ms d'Oxford 151. Voir aussi Mekhilta de-rabbi Shim'on ben Yohay, éd. Epstein-Melamed, p. 81.

96. Ce raisonnement peut être rapproché de l'affirmation de S. Friedman («Ṣelem demut wetabnit ", Sidra, p. 91) : la Bible et la littérature talmudique parlent de la forme (demut) de Dieu et non de son corps (guf).

97. Talmud Babli, Sanhedrin, 38b.

98. G. Stroumsa, «Forme(s) de Dieu : Métatron et le Christ », p. 75.

99. G. Stroumsa, « Polymorphie divine et transformations d'un mythologème, l'Apocryphon de Jean et ses sources ", in Savoir et salut, p. 59.

100. G. Stroumsa, op. cit., p. 46 et 57-58.

101. M. Idel, Ben : Sonship and Jewish Mysticism, p. 119-148.

102. Sur ce point, voir G. Stroumsa, «Polymorphie divine », p. 75, 79 et 81. Dans cette étude, la question de l'incarnation n'est cependant abordée que de manière périphérique.

103. Voir (entre autres) A. Goshen Gottstein, «The Body as Image of God », p. 192.

104. Le rapprochement entre Métatron et la Shekhina est une donnée ancienne de l'érudition, comme le montre l'étude de George F. Moore, «Intermediaries in Jewish Theology - Memra, Shekhinah, Metatron », Harvard Theological Review, 15, 1922, p. 41-85.

105. Voir E. Urbach, Les Sages d'Israël, p. 46-47.

106. Voir notre développement sur la Shekhina dans le 3) de la présente étude. Pour E. Urbach, en revanche, la Shekhina n'est pas une hypostase (Les Sages d'Israël, p. 69).

107. L'absence de précision des rabbins sur cette question s'explique certainement par une conjonction de facteurs (caractère ésotérique de la nature du corps divin, théologie manquant de rigueur conceptuelle...). 
108. Sur le trouble que suscitait ce paganisme zoomorphe chez ses observateurs grecs et romains, voir Gideon Bohak, "The Ibis and the Jewish Question: Ancient "Anti-Semitism" in Historical Context ", in Menachem Mor, Aharon Oppenheimer, Jack Pastor et Daniel R. Schwartz, Jews and Gentiles in the Holy Land in the Days of the Second Temple, the Mishnah and the Talmud, Jérusalem, 2003, p. 36-37.

109. Richard Kalmin, «Idolatry in Late Antique Babylonia », chap. 5 de Jewish Babylonia between Persia and Roman Palestine, Oxford, 2006, p. 103-120.

110. Op. cit., p. 109 et ss. R. Kalmin s'oppose à l'idée défendue par E. Urbach et S. Lieberman, selon laquelle l'idolâtrie avait perdu tout attrait pour les juifs. Pour une position proche de celle de R. Kalmin, voir Mireille Hadas-Lebel, «Le paganisme à travers les sources rabbiniques", in Aufstieg und Niedergang der römischen Welt, II, $19: 2$, Berlin, New York, 1979, p. 398 et Emmanuel Friedheim, Rabbinisme et paganisme en Palestine romaine. Étude historique des Realia talmudiques ( $I^{\text {er }}-I V$ ${ }^{e}$ siècles), Leyde, 2006.

111. Voir Arnobe de Sicca, Contre les Gentils, III, 12 : les païens reprochent aux chrétiens d'avoir aussi des conceptions idolâtres. Arnobe rétorque que ce sont les juifs qui croient, comme les païens , en un Dieu corporel. Voir également Y. Lorberbaum (Șelem Elohim, p. 20) : les païens utilisent des images dans une finalité théurgique et dans la théurgie rabbinique, c'est l'homme, image de Dieu, qui joue un rôle semblable.

112. Wa-yiqra Rabba, 34, 3, ms de Londres $340: 2$. Sur la compréhension anthropomorphique du terme selem et le culte de l'empereur, voir Morton Smith, "The Image of God: Notes on the Hellenization of Judaism with Especial Reference to Goodenough's Work on Jewish Symbols », Bulletin of the John Ryland Library, 40, 1958, p. 473-512.

113. Mekhilta de-Rabbi Yishma'el, Yitro, Ba-hodesh, 8, ms d'Oxford 151.

114. Bereshit Rabba, 68, 12, ms de Vatican 30.

115. Voir S. Friedman, « Graven Image », Graven Images : A Journal of Culture, Law and the Sacred, I, 1994, p. 233-238 ; «Ṣelem demut we-tabnit», Sidra, p. 118-133 ; «Anthropomorphism and its eradication », p. 168-178.

116. Bereshit Rabba, 39, 11 .

117. Midrash Hallel, p. 88.

118. Midrash Tanhuma, Yitro, 11 et Ephraïm Urbach, "The Rabbinical Laws of Idolatry in the Second and Third Centuries in the Light of Archaeological and Historical Facts ", in Collected Writings in Jewish Studies, Jerusalem, 1999, p. 176-177.

119. Homélies Pseudo-clémentines, 11, 4 (trad. A. Siouville, p. 244).

120. Midrash Hallel, p. 99.

121. Debarim Rabba, éd. S. Lieberman, p. 65.

122. Talmud Babli, 'Aboda Zara, $43 b$.

123. Sur la théorie du reflet, voir A. Goshen Gottstein, op. cit., p. 188.

124. Un texte de la Mekhilta (Mekhilta de-Rabbi Yishma'el, Yitro, Ba-hodesh, 6) mentionne toutes les représentations idolâtres interdites. Or il est frappant de constater dans la liste l'absence de l'homme.

125. L'homme est régulièrement considéré comme une créature plus importante que l'ange par les rabbins. Voir sur ce motif: Peter Schäfer, Rivalität zwischen Engeln und Menschen. Untersuchungen zur rabbinischen Engelvorstellung, Berlin-New York, 1975.

126. Shemot Rabba, $43,8$.

127. Voir Saul Lieberman, Yewanit wi-Ywanut, Jérusalem, 1962, p. 250-251.

128. Sur Hélios, voir Gideon Bohak, Ancient Jewish Magic. A History, Cambridge, 2008, p. 251-253.

129. Voir sur ce point: Y. Eliav, "Viewing the Scriptural Environment », p. 411-433 ; M. HadasLebel, «Le paganisme à travers les sources rabbiniques », p. 397-489; Pierre Prigent, Le Judaïsme et l'Image, Tübingen, 1990 ; Seth Schwartz, «Gamaliel in Aphrodite's Bath. Palestinian Judaism and Urban Culture in the Third and Forth Centuries", in Peter Schäfer (éd.), The Talmud 
Yerushalmi and Graeco-Roman Culture, t. I, Tübingen, 1998, p. 203-217 ; E. Urbach, « The Rabbinical Laws of Idolatry ", p.151-193. Curieusement, les magiciens sont parfois plus stricts que les rabbins et plus fidèles à l'époque du second Temple, sur le plan de l'iconophobie (G. Bohak, Ancient Jewish Magic, p. 347).

130. Mishna, 'Aboda Zara, $3,1$.

131. Y. Eliav, "Viewing the Scriptural Environment», p. 423. De nombreux commentateurs voient plutôt dans cette mishna une allusion aux statues de l'empereur qui seraient donc plus stigmatisées que d'autres formes de statues (E. Urbach, "The Rabbinical Laws of Idolatry", p. 177 ; Mireille Hadas-Lebel, Jérusalem contre Rome, Paris, 1990, p. 328-329).

132. Mishna, 'Aboda Zara, 3, 4.

133. Talmud Yerushalmi, Shebi'it, 8, 8 .

134. Voir E. Urbach, "The Rabbinical Laws of Idolatry », p. 164-166 et particulièrement le texte de Shemot Rabba, 15, 17.

135. Jésus est Dieu rendu visible comme corps et comme nom. Voir aussi la position de M. Idel sur la question de l'incarnation (Ben: Sonship and Jewish Mysticism, p. 57-69).

136. D. Boyarin, « De/Re/constructing Midrash », in Carol Bakhos (éd.), Current Trends in the Study of Midrash, Leyde-Boston, 2006, p. 314. Dans la note 47, il renvoie à l'article d'A. Goshen Gottstein avec une appréciation positive.

137. Voir, D. Boyarin, « The Eye in the Torah », p. 12 et 18.

138. Voir D. Boyarin, "The Eye in the Torah», p.13-14. Sur ce point, voir également E. R. Wolfson, Through a Speculum that Shines, p. 42.

139. Pesiqta de-rab Kahana, 4,4 et Pesiqta Rabbati, 14. Voir aussi Bereshit Rabba, 27, 1.

140. E. R. Wolfson, Through a Speculum that Shines, p. 36-40.

141. Elliot R. Wolfson, "Judaism and Incarnation : The Imaginal Body of God», in Tivka FrymerKensky, David Novak, Peter Ochs, David F. Sandmel, Michael A. Signer (éd.), Christianity in Jewish Terms, Boulder-Oxford, 2000, p. 239-254.

142. G. Stroumsa, op. cit., p. 68-70. On peut cependant se demander si le processus n'est pas plus « simple » : un christianisme qui s'éloigne progressivement de ses racines juives, ne va-t-il pas se rapprocher des pratiques « idolâtres » des païens?

\section{RÉSUMÉS}

Des travaux récents et de plus en plus nombreux ont remis en question une idée fortement établie dans le monde traditionnel juif mais aussi dans celui de l'étude scientifique des textes bibliques ou talmudiques, celle de l'incorporéité divine. Le présent article est centré sur les textes des rabbins de l'Antiquité ( $\mathrm{I}^{\mathrm{er}}-\mathrm{V}^{\mathrm{e}}$ siècles), traitant de la question du corps de Dieu. Ces textes ont suscité des débats et continuent d'en susciter. Comment faut-il lire un texte anthropomorphique? Si Dieu a un corps pour les rabbins, quelle est la nature de ce corps? Si le Dieu du judaïsme antique est corporel, quelle relation entretient-il avec la notion païenne ou chrétienne de la divinité, qui comporte aussi une facette corporelle?

An ever-increasing number of recent studies are contesting a notion that is very widely accepted in traditional Judaism and also in Biblical and Talmudic scholarship : divine incorporeity. This paper deals with ancient rabbinic texts (0-500 CE) on the question of God's body. These texts have given and continue to give rise to many controversies. How should we read an anthropomorphic 
text? If God indeed has a body according to the rabbis, what is the nature of this body? If the God of ancient Judaism is a corporeal being, how is this corporeity related to the Christian or pagan notion of divinity, which also contains a corporeal aspect?

\section{AUTEUR}

JOSÉ COSTA

Université Paris III - Sorbonne Nouvelle

jose.costa30@aliceadsl.fr 